\title{
A manipulative activity for exploring effective nuclear charge $\left(Z_{\text {eff }}\right)$
}

Jillian Kasman^, Taylor Slouka^, Alan L. Kiste*, Javin P. Oza*

Department of Chemistry and Biochemistry, California Polytechnic State University, San Luis Obispo, CA, 93407, USA

${ }^{\wedge}$ Authors contributed equally.

\section{ABSTRACT}

High-school and undergraduate college students may often rely on memorization when learning periodic trends, leading to an ineffective understanding of introductory chemistry concepts like atomic radius, ionization energy, and electron affinity. Comprehension of effective nuclear charge $\left(Z_{\text {eff }}\right)$ is foundational to a complete understanding of periodic trends. $Z_{\text {eff }}$ remains an abstract concept for many students, indicating that a manipulative activity for teaching the phenomenon could be a useful way to explain the concept for greater student comprehension. Here we report a hands-on learning activity to target this knowledge gap using magnetic attraction as an analogy for electrostatic attraction within the atom. This approach enables students to explore the phenomenon of effective nuclear charge at a macroscopic level and apply their learning to periodic trends and related concepts. We anticipate that this activity will fill a long-standing hole in the active learning of chemical principles. Importantly, this activity is low-cost and can be assembled using readily accessible materials to allow implementation in most classrooms and virtual learning environments.
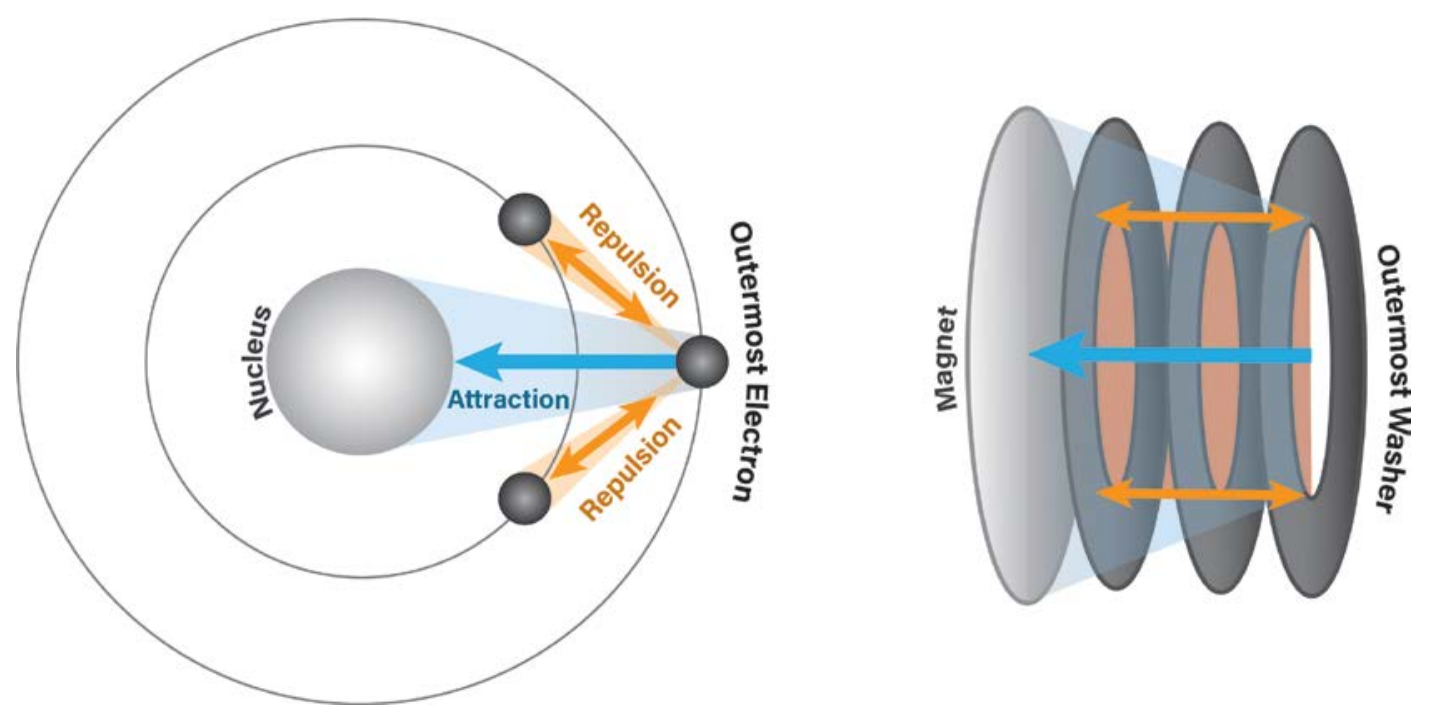

\section{KEYWORDS}

Effective Nuclear Charge, $Z_{\text {eff }}$, High School Chemistry, Introductory Chemistry, First-Year Undergraduate, General Chemistry, Hands-On Learning/Manipulatives, Atomic Structure 
As an abstract idea in chemistry, effective nuclear charge may be difficult to comprehend in a classroom setting. Many high school and college textbooks ${ }^{1-3}$ provide accurately written explanations of effective nuclear charge but lack clear visual representations that are vital for student comprehension. Textbook explanations often utilize the equation:

$$
Z_{\text {eff }}=Z-\sigma
$$

where $Z_{\text {eff }}$ is the effective nuclear charge, $Z$ is the nuclear charge, and $\sigma$, the shielding constant, is calculated based on the electrons in shells and subshells between and among valence electrons and the nucleus. ${ }^{1}$ Relying on a mathematical description rather than a conceptual explanation may not provide students with the relevant atomic-level understanding of the phenomenon. Many textbooks ${ }^{4-7}$ and published works ${ }^{22,23}$ may emphasize visual illustrations of periodic trends, such as that seen in Figure 1, but lack descriptive explanations of the atomic-level structure-property relationships that explain those trends. Given that a conceptual understanding of effective nuclear charge is crucial for fully comprehending periodic trends, students who fail to understand the concept may rely on memorization and heuristics.

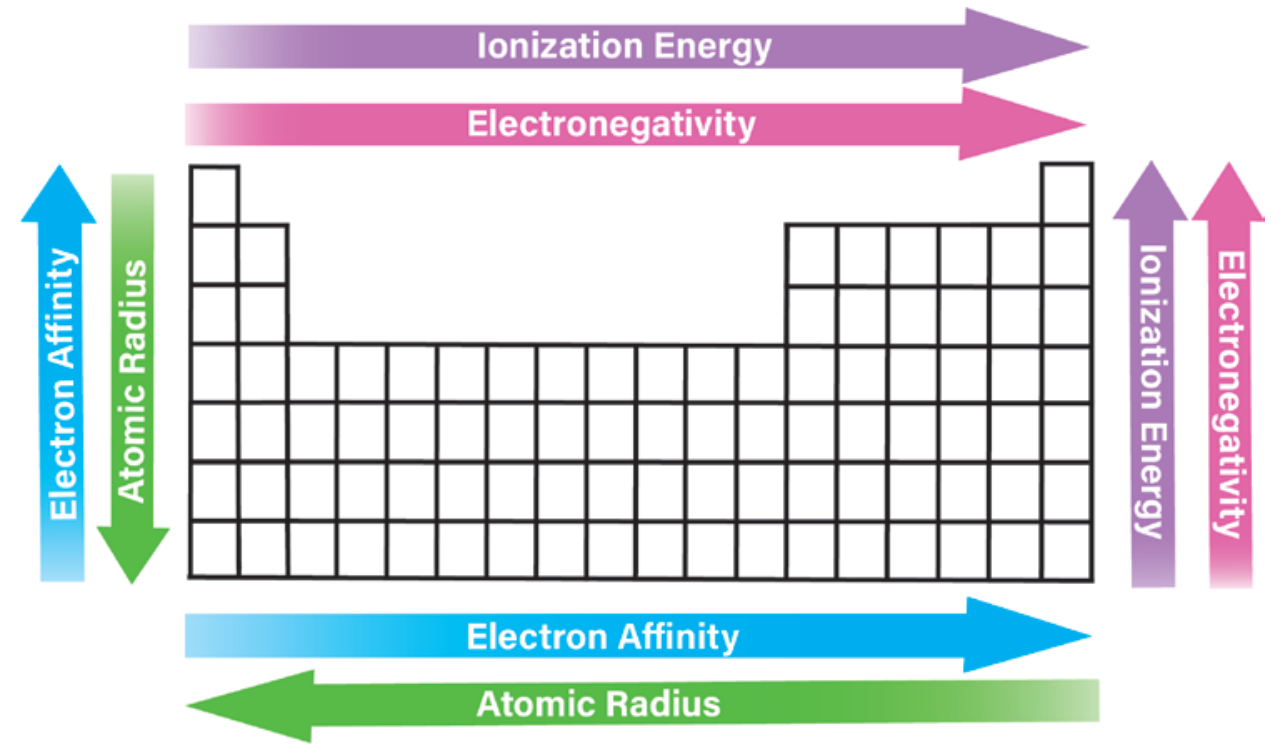

Figure 1. Illustration of the periodic trends commonly displayed in introductory Chemistry textbooks ${ }^{4-7}$. Therefore, when asked to explain the atomic-level basis for periodic properties of elements, such as 'why does fluorine have a higher electron affinity than chlorine?', we have observed that students often 
respond that fluorine is higher on the periodic table than chlorine, which, while true, is an answer based on a heuristic rather than on an understanding of a physical model of the atom.

The lack of a thorough comprehension of the atomic-level explanation for the origin of effective nuclear charge may lead to misconceptions that negatively impact student mastery of later curricula. Examples of such misconceptions include the assumption that increasing atomic numbers directly correlates to increasing atomic radius or that atoms with the greatest electron affinity also have the highest electronegativity. ${ }^{8}$ A possible underlying reason for these misunderstandings about periodic trends is a limited understanding of the concept of effective nuclear charge. ${ }^{9-10}$

Educational manipulatives that provide a hands-on analogy and the use of representations to explain effective nuclear charge could reduce common misconceptions. Developing representational competence allows students to translate a visualization of a chemical idea or process into logical understanding. ${ }^{1-13}$ Manipulatives are a common tool in chemistry used to develop these skills and simplify a variety of abstract concepts using physical representations. ${ }^{14-16,}{ }^{24}$ Despite the important role manipulatives play in chemistry education, there remains a lack of options that provide a physical representation as an analogy for effective nuclear charge.

By working with a tangible portrayal of the phenomena, students are encouraged to take an analytical approach, thus increasing critical thinking and communication skills. ${ }^{17}$ Specifically, the use of motor skills adds kinesthetic detail to student thinking and activates the sensorimotor brain system, an area not activated when simply reading or listening. Activation of this region has been shown to improve understanding and performance in science education. ${ }^{14}$ Active learning, an instructional approach that promotes student engagement in the learning process, has also been shown to benefit student attitudes, understanding, and thinking skills.18-19 One method that promotes active learning is the integration of activities into traditional lectures. By working through activities, students are more likely to remember key concepts and avoid misconceptions. ${ }^{17,}{ }^{20}$ An active learning approach may also aid students in forming representational fluidity, vital for applying understanding of effective nuclear charge to related topics. Therefore, the goal of this activity is to shift away from memorization-focused instruction in favor of a comprehensive explanation that will increase accurate and thorough student knowledge. 
Focusing on a physical analogy/representation for effective nuclear charge can also provide an entry point to explanations of related concepts like ionization energy, atomic radii, and electron affinity that are essential to general chemistry understanding.

This effective nuclear charge activity offers an active learning approach to the concept by using magnetic attraction as a proxy for electrostatic interactions within the atom. Using low-cost and readily available materials, this lesson is easily replicated in high-school or college-level general chemistry courses to help students develop their chemical intuition and apply what they learn to other phenomena. Using a flat, circular magnet to represent the positively charged nucleus and flat washers to represent electrons, students will be able to investigate effective nuclear charge and use their new understanding of the concept to make causal explanations of periodic trends.

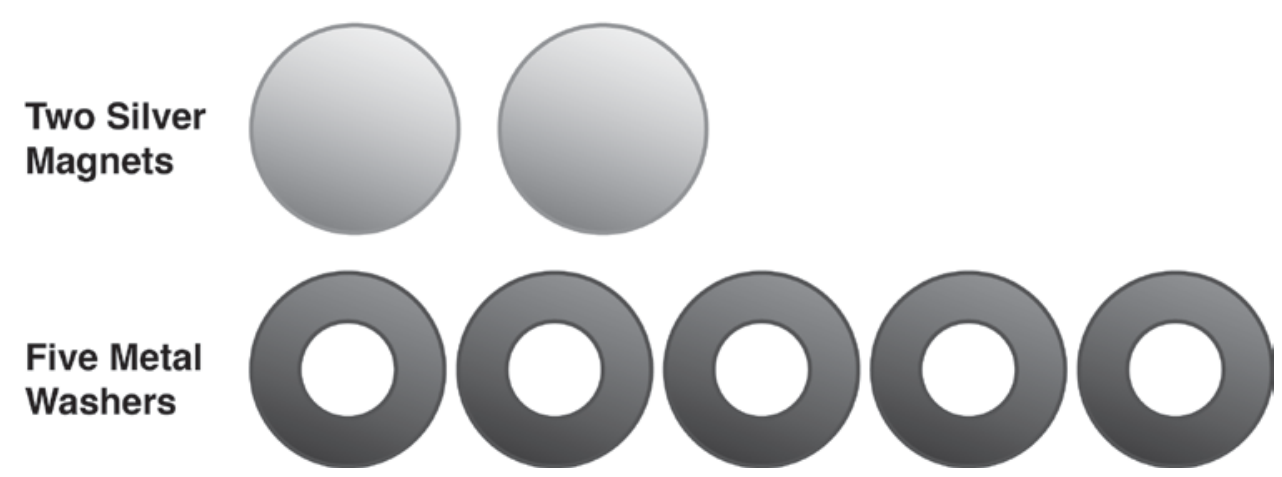

Figure 2. Materials needed to carry out the effective nuclear charge classroom activity. Two identical silver magnets represent the protons $(Z)$ in the nucleus of an atom, whereas five metal washers represent the electrons of that same atom.

This activity has been pilot tested for our first quarter of general chemistry courses in our on-campus studio classrooms, which combine lecture and laboratory teaching to provide a variety of hands-on and other active learning opportunities. ${ }^{21}$ Students worked through the lesson collaboratively in clusters of eight. However, if desired by an instructor, students could work individually or in teams of 2-4 students to enhance communication and collaboration skills. The components are inexpensive and straightforward, allowing 
instructors to produce kits for many different learning environments including large lectures, student materials is included in the Supporting Information.

Each student is provided with a kit containing two silver magnets and five metal washers (Figure 2). First, students investigate the change in the magnetic attraction between the magnet (nucleus) and the outermost washer (valence electron) by observing how the number of washers (inner electrons) added changes the difficulty of removing the outermost washer. Students will find that as more washers are added, it becomes increasingly easier to remove the outermost washer, implying a decrease in attraction (Figure 3). This decrease in attraction, in the context of the comparison made in this activity, suggests an increase in shielding as the number of electrons in an atom increases. Next, students explore how adding a second magnet changes the the difficulty of removing the outermost washer. Students will find that as the number of magnets increases, the attraction between the magnet and the outermost washer increases, demonstrating increased attraction between the nucleus and electrons for atoms with a larger number of protons (Figure 4).

Students are then told that the magnets represent the number of protons $(Z)$ and the outermost washer represents the valence shell electrons of that atom, thus encouraging students to analyze the connection between magnetic and electrostatic attraction. Several questions are then posed to the students, helping them connect their observations to the concept of effective nuclear charge and asking them to infer general relationships about the number of protons in the nucleus, the number of inner electrons, and the attraction and repulsion forces on the valence electrons. Lastly, students are asked to consider the limitations of this analogy in representing the true relationship between electrons and 
protons within an atom to make clear that electrons repel each other, unlike like the washers in this activity.
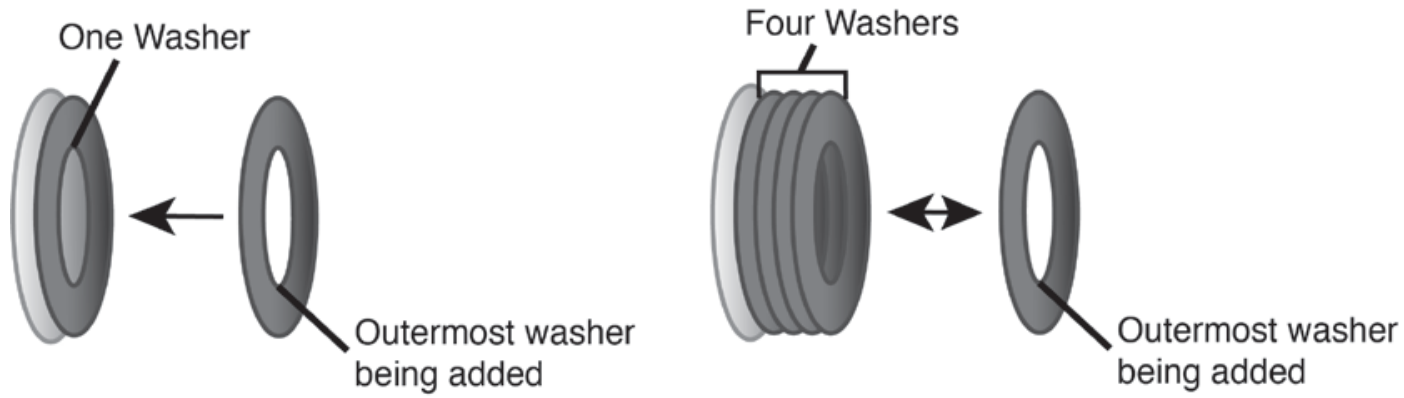

Figure 3. Layering additional washers onto one magnet decreases attraction between the magnet and outermost washer. This demonstrates how increased shielding decreases the effective nuclear charge for electrons in an atom.
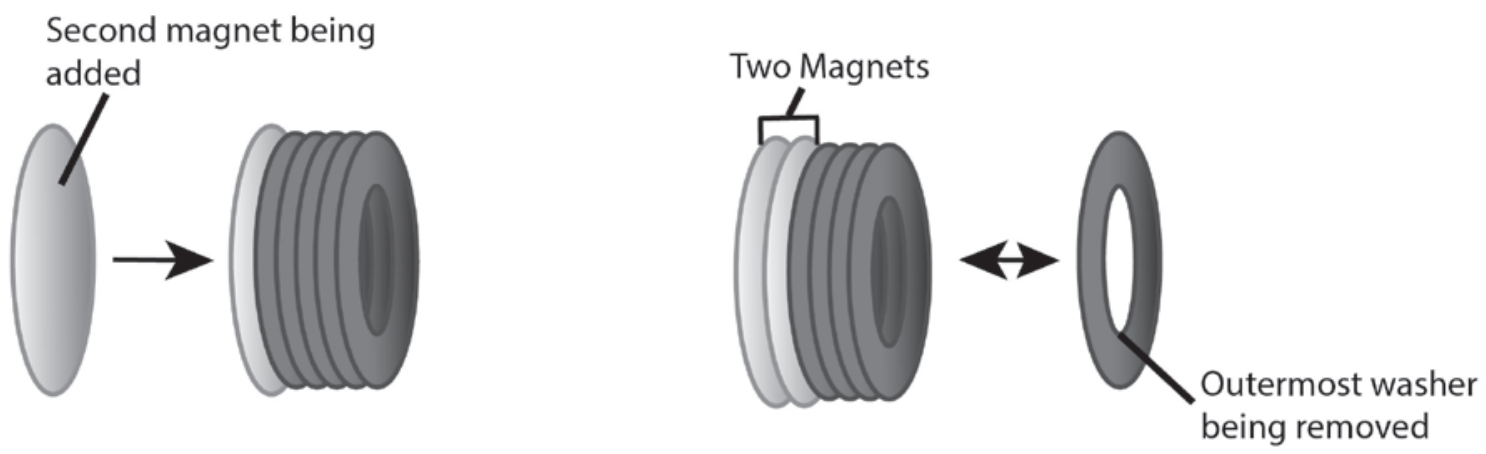

Figure 4. The outermost washer is harder to remove when an additional magnet is added. This demonstrates how an increase in the number of protons $(Z)$ will increase the effective nuclear charge experienced by electrons within that atom.

Through this activity, students can assess the implications of effective nuclear charge on periodic trends such as atomic radius, ionization energy, and electron affinity. Students can observe that a change in attraction between the magnet and outermost washer correlates to a change in effective nuclear charge for a valence electron. Using this manipulative for the concept, students can infer that atoms with a larger number of protons or lack of shielding lead to a smaller atomic radius, increased ionization energy, and increased electron affinity. By allowing students to create a mental model for effective nuclear charge, we believe they 
will be able to provide more effective causal explanations for periodic trends, rather than simply relying on memorization and heuristics.

This $Z_{\text {eff }}$ activity was implemented in one section of our first-quarter general chemistry studio classroom serving 64 students in Fall 2019. Six weeks later, these students, along with all 559 students enrolled in all sections of the course, took a common final exam in which they were asked the question "How does effective nuclear charge $\left(Z_{\text {eff }}\right)$ affect average atomic size?" Students were presented with multiple-choice options relating how "tightly" the electrons are "held" to increasing or decreasing average atomic size. $82.5 \%$ of the 64 students who experienced the manipulative activity answered correctly compared to $74.5 \%$ of the entire population of students who answered correctly. The persistence of the learning gain in this preliminary assessment provides the impetus for a more thorough investigation of representational competence of effective nuclear charge.

\section{SUMMARY}

We have introduced a hands-on activity for effective nuclear charge that uses magnetic attraction as an analogy for the relationship between the nucleus and electrons within an atom. Students use magnets to represent nuclear attraction and washers to represent electrons. This allows students to develop mental models for effective nuclear charge, potentially avoid common misconceptions associated with the phenomena, have higher engagement, and help build critical thinking skills. The analogy introduced in this activity can be used for the understanding of related concepts such as atomic radius, electron affinity, ionization energy, and other periodic trends.

\section{ASSOCIATED CONTENT}

Supporting Information

The Supporting Information containing activity instructions and worksheet is available on the ACS Publications website at DOI: 10.1021/acs.jchemed.XXXXXXX. [ACS will fill this in.] Example brief 


\section{AUTHOR INFORMATION}

Corresponding Authors

*E-mail: akiste@calpoly.edu; joza@calpoly.edu

\section{ACKNOWLEDGMENTS}

We thank Dr. Gregory Scott for his detailed feedback on this manuscript. We also thank our fellow

General Chemistry instructors for their feedback on the activity's design and implementation, especially Drs. Joanna Laird, Francesco Contu, and John Hagen who provided helpful feedback during the initial development of the activity and manuscript preparation. Our learning assistants Layne Williams, Kyle Farrell, and Kayla Hong helped with assembling the kits and assisted with classroom implementation.

\section{REFERENCES}

1. Haas, K. 1.1.2: Effective Nuclear Charge https://chem.libretexts.org/Courses/Saint_Mary's_College\%2C_Notre_Dame\%2C_IN/CHEM_3 42\%3A_Bioinorganic_Chemistry/Readings/Week_1\%3A_Analysis_of_Periodic_Trends/1.1\%3A_Concepts_a nd_principles_that_explain_periodic_trends / 1.1.2\%3A_Effective_Nuclear_Charge (accessed Jul 9, 2020).

2. Averill, B. A.; Eldredge, P. General Chemistry: Principles, Patterns, and Applications; Saylor Academy, 2012.

3. Flowers, P.; Theopold, K.; Langley, R.; Robinson, W. R. 6.5 Periodic Variations in Element Properties. In Chemistry 2e; OpenStax: Houston, Texas, 2019.

4. Bauer, R. C.; Birk, J. P.; Marks, P. S. A Conceptual Introduction to Chemistry, 1st ed.; McGrawHill Higher Education, 2007.

5. Silberberg, M. Chemistry: The Molecular Nature of Matter and Change, 5th ed.; McGraw-Hill Publishing Company, 2009.

6. Sochocki, J. Conceptual Chemistry, 3rd ed.; Prentice Hall, 2006.

7. Zumdahl, S.; Zumdahl, S. Chemistry, 8th ed.; Brooks Cole: Belmont, CA, 2010.

8. Hanson, R.; Kwarteng, T. A.; Antwi, V. Undergraduate Chemistry Teacher Trainees' Understanding of Chemical Phenomena. European Journal of Basic and Applied Sciences 2015, $2(3), 7$.

9. Shenk, Lynne. Student Reasoning Strategies Concerning Periodic Trends. Ph.D. Dissertation, University of Minnesota, 2018. 
10. Salame, I.; Sarowar, S.; Begum, S.; Krauss, D. Students' Alternative Conceptions about Atomic Properties and the Periodic Table. Chem. Educator 2011, 16, 190-194.

11. Daniel, K. L.; Bucklin, C. J.; Austin Leone, E.; Idema, J. Towards a Definition of Representational Competence. In Towards a Framework for Representational Competence in Science Education; Daniel, K. L., Ed.; Models and Modeling in Science Education; Springer International Publishing: Cham, 2018; pp 3-11. https://doi.org/10.1007/978-3-319-899459.1.

12. Kozma, R., Chin, E., Russell, J., \& Marx, N. The Roles of Representations and Tools in the Chemistry Laboratory and Their Implications for Chemistry Learning. Journal of the Learning Sciences 2000, 9 (2), 105-143.

13. Kozma, R.; Russell, J. Students Becoming Chemists: Developing Representational Competence; Springer: Dordrecht, 2005; Vol. 1. https://doi.org/10.1007/1-4020-3613-2_8. Learning. Psychol Sci 2015, 26 (6), 737-749. https://doi.org/10.1177/0956797615569355.

15. Ruddick, K. R.; Parrill, A. L. JCE Classroom Activity \# 113: An Interlocking Building Block Activity in Writing Formulas of Ionic Compounds. J. Chem. Educ. 2012, 89 (11), 1436-1438.

16. Kiste, A. L.; Hooper, R. G.; Scott, G. E.; Bush, S. D. Atomic Tiles: Manipulative Resources for Exploring Bonding and Molecular Structure. J. Chem. Educ. 2016, 93 (11), 1900-1903. https://doi.org/10.1021/acs.jchemed.6b00361.

17. Shatri, K.; Buza, K. The Use of Visualization in Teaching and Learning Process for Developing Critical Thinking of Students. Eur. j. soc. sci. educ. res. 2017, 4 (1), 71. https://doi.org/10.26417/ejser.v9i1.p71-74.

18. Mathias, A. Active Learning in the Science Classroom. 2014, 33.

19. Bobek, E.; Tversky, B. Creating Visual Explanations Improves Learning. Cogn Res Princ Implic 2016, 1. https://doi.org/10.1186/s41235-016-0031-6.

20. Freeman, S.; Eddy, S. L.; McDonough, M.; Smith, M. K.; Okoroafor, N.; Jordt, H.; Wenderoth, M. P. Active Learning Increases Student Performance in Science, Engineering, and Mathematics. Proceedings of the National Academy of Sciences 2014, 11 (23), 8410-8415. https://doi.org/10.1073/pnas.1319030111.

21. Kiste, A. L. Implementing the Studio Classroom in Chemistry. In Active Learning in College Science: The Case for Evidence-Based Practice; Mintzes, J. J., Walter, E. M., Eds.; Springer International Publishing: Cham, 2020; pp 521-540.

22. A Visually Attractive "Interconnected Network of Ideas" for Organizing the Teaching and Learning of Descriptive Inorganic Chemistry Glen E. Rodgers* J. Chem. Educ. 2014, 91, 2, 216-224 https://doi.org/10.1021/ed3003258 
23. Periodic Universe: A Teaching Model for Understanding the Periodic Table of the Elements Matthias Bierenstiel* and Kathy Snow Journal of Chemical Education 2019, 96, 7, 1367-1376 (Article)

DOI: $10.1021 /$ acs.jchemed.8b00740

24. Measuring the Force between Magnets as an Analogy for Coulomb's Law Samuel P. Hendrix and Stephen G. Prilliman* Journal of Chemical Education 2018, 95, 5, 833-836 (Demonstration)

DOI: $10.1021 /$ acs.jchemed.7b00580 25. 